\title{
Crystallization and Melting of Binary Mixtures of Nylon 6 and Nylon 66. A Study by DSC
}

\author{
Tsunetoshi Matsuda, Takehiko Shimomura, ${ }^{*}$ and Matsuo Hirami ${ }^{\dagger}$ \\ Research and Development Center, Unitika Ltd., \\ 23 Kozakura, Uji, Kyoto 611-0021, Japan \\ * Unitika Research Laboratories Inc., \\ 23 Kozakura, Uji, Kyoto 611-0021, Japan
}

(Received January 18, 1999)

\begin{abstract}
The crystallization and melting behavior of binary mixtures of nylon 6 and nylon 66 was investigated by differential scanning calorimetry (DSC). Mixture samples prepared from solution were carefully arranged so as to attain a well-mixed state prior to the measurement of DSC. In the mixtures nylon 6 and nylon 66 crystallize separately; nylon 66 crystallizes preferably, while the crystallization of nylon 6 was restricted remarkably, especially in the region where the content of nylon 6 was small. In melting temperature diagram, considerable reduction of melting temperature of nylon 66 was observed, probably due to the diluent effect caused by nylon 6 . Analysis of this reduction in terms of the Nishi-Wang equation made it possible to estimate the polymer-polymer interaction parameter for the present mixture system. Obtained parameter is a relatively large negative value and the contribution from the entropy term is significant. Melting temperature of nylon 6 in the mixtures was observed in the narrow range of composition where the content of nylon 66 is small. In the crystallization temperature diagram, the crystallization temperature read from the peak position of the crystallization profile starts from nylon 66, decreased in the mixtures and leveled off. Total crystallinity including both nylon components evaluated from the area under the profile decreased appreciably in the intermediate region of composition. The slope of increasing curve of each profile on cooling process was evaluated as a relative measure for the rate of crystal growth. The composition dependence of the slope thus obtained is discussed qualitatively from the viewpoint of crystallization kinetics concerning crystal growth of both nylon components in the mixtures.
\end{abstract}

KEY WORDS Binary Mixtures / Crystallization / Crystallization Temperature / Degree of Crystallinity / Differential Scanning Calorimetry / Melting Temperature Depression / Nylon 6 / Nylon 66 / Polymer Blends / Polymer-Polymer Interaction Parameter /

This and subsequent papers ${ }^{1,2}$ will be concerned with the crystallization of binary blends of nylon 6 and nylon 66. The crystallization and melting of binary mixtures involving a crystalline polymer component, i.e., (a) polymer-low molecular weight diluents and (b) mixtures of a crystalline-amorphous polymer pair, have been extensively studied. ${ }^{3-6}$ In contrast to the comparatively advanced knowledge on binary mixtures of type (a) and type (b), progress as a whole toward understanding of the features of (c) binary mixtures consisting of two crystalline polymers seems insufficient. Thus, it is desirable to provide further information useful for the elucidation of the phase behavior of binary mixtures of type (c). Even in this field, the crystallization and phase structures of binary blends of two different polyethylenes have been investigated extensively, especially by Stein $e t$ $a l .{ }^{7}$ and Mandelkern et al. ${ }^{8}$ In their studies, attention was directed the possibility of cocrystallization and conditions of the cocrystallization were determined by the molecular features of the chain, namely branching concentration and whether the sample was subjected to slow or rapid crystallization. This investigation is confined to binary mixtures systems of two crystalline polymers, where both components are miscible and crystallize separately. This requirement will be probably satisfied in the case of mixtures of nylon 6 and nylon 66.

Nylon 6 and nylon 66 are polyamides widely used as industrial products and information of their behavior in blends is interesting. However, reports of investigations in this area are few. ${ }^{9,10}$ Recently, Rybnikar and Geil ${ }^{11}$ presented an extensive investigation of crystallization, melting and recrystallization of binary blends of nylon 6 and nylon 66 with a special attention on interactions at phase boundary of the respective polymer components. The work described here was undertaken to provide further information to understand the phase behavior of mixtures of nylon 6 and nylon 66 systematically over the whole range of composition.

This work measures differential scanning calorimetry (DSC) for binary mixtures of nylon 6 and nylon 66 carefully prepared so as to attain a well-mixed state in the melt prior to the measurement of DSC. From DSC measurements of the mixture samples on heating and cooling, (i) higher and lower melting temperaturecomposition relations and (ii) variation in crystallization profile over the whole range of composition have been obtained. The phase behavior of this binary system of nylon 66 has been examined from thermodynamics and crystallization kinetics.

\section{EXPERIMENTAL}

\section{Samples}

Nylon 6 with $M_{w}=16000$ (Unitika Ltd.; A 1030BRL) and nylon 66 with $M_{w}=22000$ (BASF Co.; R8270) were used to prepare the mixture samples. Pellets of corresponding ratio were well dissolved in formic acid, and the solutions were flashed into excess methanol. The precipitated powder is filtered and dried for the use of DSC measurements.

\footnotetext{
$\uparrow$ To whom correspondence should be addressed.
} 


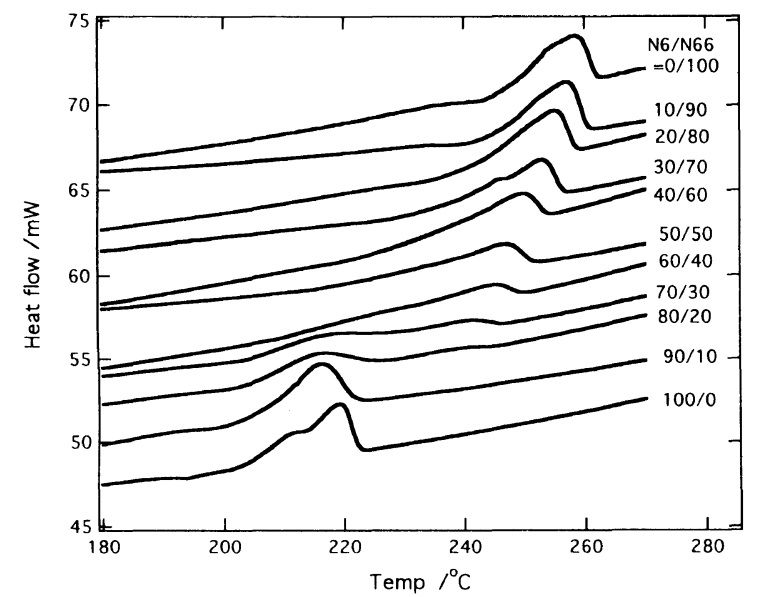

Figure 1. Melting diagram for mixtures of nylon 6 and nylon 66 obtained in the heating measurement. Curves are shifted vertically for the clarification.

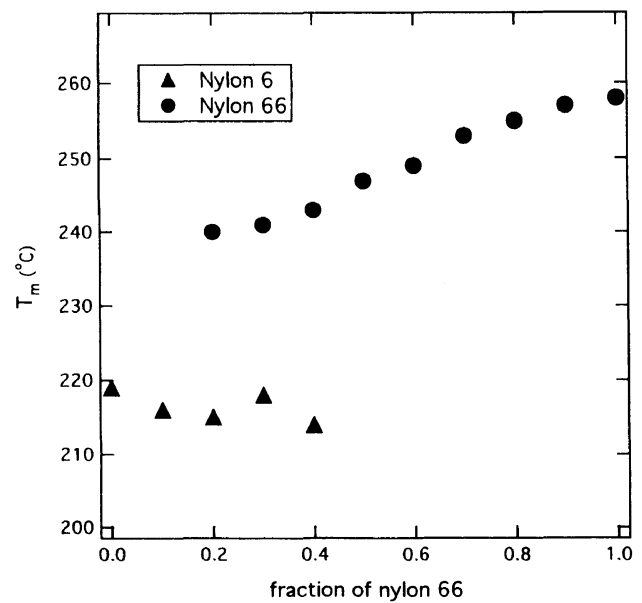

Figure 2. Relation between the melting temperatures and the composition of the mixtures of nylon 6 and nylon 66 .

\section{Measurement}

Measurements of the melting and crystallization profile were carried out by the Perkin-Elmer DSC 2B differential scanning calorimeter. A sample of $c a .5 \mathrm{mg}$ was filled in a measurement cell of DSC and the temperature was first elevated to $280^{\circ} \mathrm{C}$ by $500^{\circ} \mathrm{C} \mathrm{min}^{-1}$ and held for $10 \mathrm{~min}$. The sample was quenched to the room temperature by $500^{\circ} \mathrm{Cmin}^{-1}$. Heating measurement was done at the heating speed of $10^{\circ} \mathrm{C} \mathrm{min}^{-1}$ up to $280^{\circ} \mathrm{C}$ followed by the cooling measurement at $-10^{\circ} \mathrm{C} \mathrm{min}^{-1}$.

\section{RESULTS AND DISCUSSION}

\section{General Features}

The melting temperature diagram obtained for the mixtures of nylon 6 and nylon 66 is shown in Figure 1. The melting temperatures read from a peak position of endotherm against composition are plotted in Figure 2. Two melting peaks are observed for the mixtures in which nylon 66 content is smaller, while the lower-temperature melting peak disappears when nylon 6 content is less than $50 \%$.

The crystallization temperature diagram measured in the cooling process from $280^{\circ} \mathrm{C}$ at the cooling speed of $10^{\circ} \mathrm{Cmin}^{-1}$ is shown in Figure 3 , and the crystalliza-

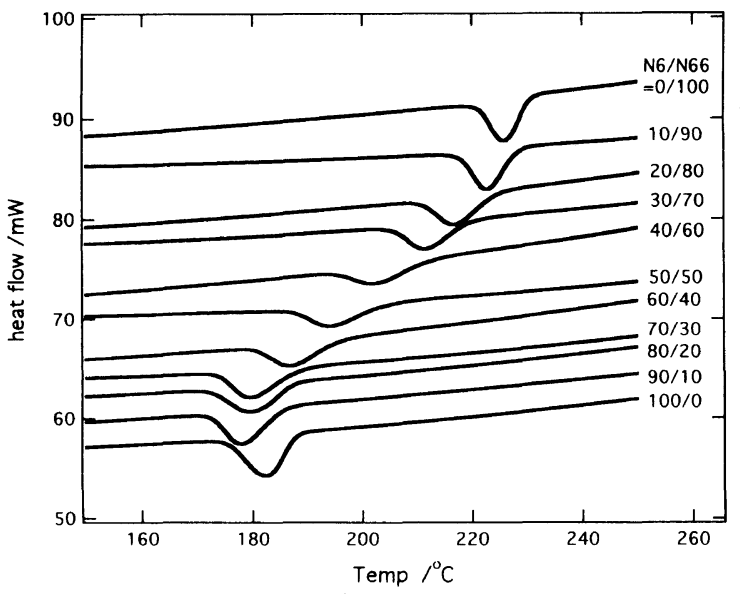

Figure 3. Crystallization diagram for the mixtures of nylon 6 and nylon 66 obtained in the cooling measurement. Curves are shifted vertically for the clarification.

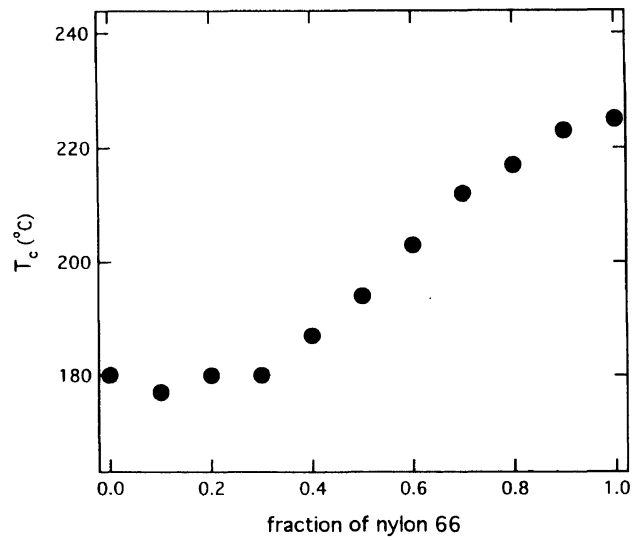

Figure 4. Relation between the crystallization temperatures and the composition of the mixtures of nylon 6 and nylon 66 .

tion temperature as the peak position of exotherm against the composition is shown in Figure 4. For all compositions, single peaks due to crystallization are observed. After the cooling measurement, we carried out the second heating measurements for mixtures with nylon 6 content of $0.8,0.7$, and 0.6 . The melting temperature diagram was identical to the first heating measurement. Nylon 6 and nylon 66 thus crystallize separately, but two endotherms coincide to a single peak. In the heating process, crystals of each component melt at rather separated temperature and two endothermic peaks are observed.

\section{Melting Temperature Diagram}

From the melting temperature diagram for binary mixtures of nylon 6 and nylon 66 as shown in Figure 1, we recognize that the melting temperature of nylon 66 decreases considerably as the content of nylon 6 increases. The reduction of melting temperature in nylon 6/nylon 66 blends reported recently by Rybnikar and Geil ${ }^{11}$ is very small compared with our data here. They mentioned that the reduction of melting temperature could be attributed to possible co-incorporation as crystal defects although the possibility of cocrystallization was denied. The different behavior for the reduction of melting temperature in the mixtures of nylon 6 and nylon 
66 as described above is attributed to the difference in the preparation of mixture samples, which results in the different level of crystalline state in each series of nylon 6/nylon 66 mixtures. The preparation of our samples was made carefully so as to realize well-mixed state in the melt prior to the measurement of DSC.

We believe that a considerable reduction of melting temperature of nylon 66 in our case was brought about by a thermodynamic reason, that is, at equilibrium between the crystalline phase of nylon 66 and the surrounding amorphous phase consisting of nylon 66 and nylon 6 , the decrease in the chemical potential of polymer unit of nylon 66 in the amorphous phase due to the free energy of mixing causes reduction of melting temperature of the crystalline phase of nylon 66 . Experimental data for the reduction of melting temperature of nylon 66 as a function of composition may thus be analyzed as follows.

In the present binary system, nylon 6 and nylon 66 are designated as the components 1 and 2. Letting $T_{\mathrm{m} 2}$ and $\phi_{2}$ represent the melting temperature and volume fraction of the component 2 , the relation of the higher melting temperature $T_{\mathrm{m} 2} v s$. composition $\phi_{2}$ can be written by the following Nishi-Wang equation ${ }^{5}$ derived for a binary mixture of a crystalline-amorphous polymer pair. Here nylon 6 functions as a diluent of amorphous polymer.

$$
\frac{1}{T_{\mathrm{m} 2}}-\frac{1}{T_{\mathrm{m} 2}^{0}}=-\left(\frac{R}{\Delta H_{\mathrm{u} 2}}\right)\left(\frac{V_{\mathrm{u} 2}}{V_{\mathrm{u} 1}}\right)\left[\left(1-\phi_{2}\right)-\chi\left(1-\phi_{2}\right)^{2}\right]
$$

where $T_{\mathrm{m} 2}^{0}$ is the melting temperature of pure polymer and $\Delta H_{\mathrm{u} 2}$ is the heat of fusion per mole of unit of the component 2, $V_{\mathrm{u} 1}$ and $V_{\mathrm{u} 2}$ are molar volumes of unit of the components 1 and 2 , and $\chi$ is the polymer-polymer interaction parameter. ${ }^{3,12}$ As an alternative expression, the parameter $\chi$ is related to the cohesive energy density $B$,

$$
\chi=B V_{\mathrm{u} 1} / R T
$$

For wider application, the polymer-polymer interaction parameter should be extended so that the parameter $\chi$ further consists of the heat and the entropy terms, and we may write,

$$
\chi=\chi_{\mathrm{H}}+\chi_{\mathrm{s}}
$$

where $\chi_{\mathbf{H}}$ and $\chi_{\mathrm{S}}$ are heat and entropy terms, respectively. The obtained results of melting temperatures of nylon 66 in nylon 6/nylon 66 mixtures may be examined using the thermodynamic relations described above. As the thermodynamic quantities of fusion for nylon 66, we apply the literature data; $T_{\mathrm{m} 2}^{0}=267^{\circ} \mathrm{C}$ and $\Delta H_{\mathrm{u} 2}=10300$ cal mol ${ }^{-1} \cdot{ }^{13}$ Further, we put $V_{\mathrm{u} 2} / V_{\mathrm{u} 1} \simeq 2$, reflecting the unit volume of each crystal. According to the usual procedure, plots of $\left[\left(1 / T_{\mathrm{m} 2}\right)-\left(1 / T_{\mathrm{m} 2}^{0}\right)\right] / \phi_{1}$ against $\left(\phi_{1} / T_{\mathrm{m} 2}\right)$, where $\phi_{1}\left(=1-\phi_{2}\right)$ is the volume fraction of the component 1, are shown in Figure 5. For reference, plots of literature data of Nishi and Wang ${ }^{5}$ for mixtures of poly(vinylidene fluoride) (PVDF) and poly(methyl methacrylate) (PMMA) are also included. In the diagram of Figure 5, if the data are plotted as a straight linear line, the heat term of the interaction parameter can be obtained from the slope of the line, while the entropy

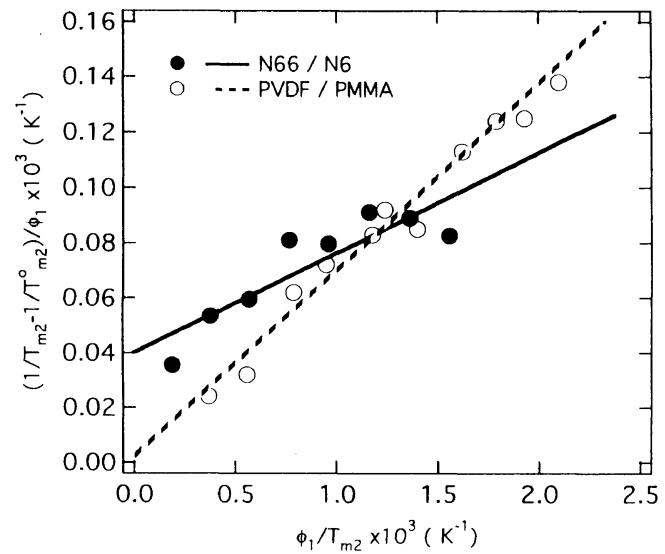

Figure 5. Plots for estimating the interaction parameters in the mixture of nylon $6 /$ nylon $66(\mathbf{O})$, and the mixture of PVDF/PMMA (O).

Table I. Estimated interaction parameters for the mixtures of nylon $6 /$ nylon 66 and PVDF/PMMA

\begin{tabular}{lcccc}
\hline & $\chi_{\mathrm{H}}$ & $\chi_{\mathrm{S}}$ & $\chi$ & $B$ \\
\hline $\begin{array}{l}\text { Nylon 6/nylon 66 } \\
\text { (present work) } \\
\text { at } 250^{\circ} \mathrm{C}\end{array}$ & -0.358 & -0.205 & -0.563 & $\mathrm{cal} /\left(\mathrm{cm}^{3}\right.$ of nylon 6) \\
\hline $\begin{array}{l}\text { PVDF/PMMA } \\
\text { (Nishi and Wang) }\end{array}$ & -0.295 & 0 & -0.295 & $\mathrm{cal} /\left(\mathrm{cm}^{3}\right.$ of PMMA) \\
at $160^{\circ} \mathrm{C}$ & & & & \\
\hline
\end{tabular}

term, from the ordinate of the intersection point. The values thus obtained together with those from literature for PVDF/PMMA mixtures are listed in Table I. For nylon 6/nylon 66 mixtures, we find the interaction parameter including both heat and entropy terms as $B=-5.06 \mathrm{cal} \mathrm{cm}^{-3}$ and $\chi=-0.563$ at $250^{\circ} \mathrm{C}$. It is interesting to compare the values with those corresponding PVDF/PMMA mixtures; $B=-2.98 \mathrm{cal} \mathrm{cm}^{-3}$ and $\chi=-0.295$ at $160^{\circ} \mathrm{C}$. A relatively large negative polymer-polymer interaction parameter indicates the characteristic of good miscibility for the given binary mixture system. This is applicable to nylon 6/nylon 66 mixtures of the present work as well as to PVDF/PMMA mixtures studied early by Nishi and Wang. ${ }^{5}$ However, these two binary systems show quite different contributions from the heat and the entropy terms to the parameter $\chi$. As can be seen in Figure 5 , in contrast to plots of PVDF/PMMA mixtures which pass the original point of the axis of coordinates very closely, plots of nylon 6/nylon 66 mixtures show a large value for the ordinate of the intersection point. The contribution of the entropy term is thus significant in nylon 6/nylon 66 mixtures. The heat and the entropy terms of $\chi$ are given by $\chi_{\mathrm{H}}=-187 / T$ and $\chi_{\mathrm{S}}=-T \times 3.92 \times 10^{-4}$. At $250^{\circ}$, we have $\chi_{\mathrm{H}}=-0.358, \chi_{\mathrm{S}}=-0.205$ and $\chi=-0.563 . \chi$ and $B$, together with the literature data for PVDF/PMMA by Nishi and Wang are listed in Table I. The unique tendency of this remarkable contribution of the entropy term is attributed to the fact that the hydrogen-bonding of intermolecular interaction plays an important role in the miscibility for nylon 6/nylon 66 mixtures. For further clarification, similar experiments for other different types 
of nylon mixtures are being done at this laboratory now, in the hope of obtaining information as to how the entropy term contribution to the $\chi$-parameter depends on the molecular characteristics of polyamide chains of both components such as the ratio of the number of amide units to that of methylene units.

As shown in Figure 1, in the melting temperature diagram where the content of nylon 66 is small, the melting temperature profile of nylon 6 can be observed at $\phi_{2}=0.1,0.2$, and 0.3 , although the peak height decreases as $\phi_{2}$ increases, and observed as a shoulder at $\phi_{2}=0.4$. For mixture samples prepared in this composition range, the coexistence of the separately formed crystalline phases of both components of nylon 6 and nylon 66 can be thus realized. In the region where the content of nylon 6 is less than $50 \%$, the crystallization of nylon 6 is extremely restricted, probably due to the predominance of the preferably formed crystalline phase of the higher melting temperature component, nylon 66 .

In Figure 3, the melting temperature of nylon 6 in nylon $6 /$ nylon 66 mixtures, read from the peak position of melting profile in DSC diagram, is plotted against composition. The melting temperature of nylon 6 seems to decrease gradually as the content of nylon 66 increases. Analysis of this behavior will be performed in a separate paper $^{14}$ in which the lower melting temperaturecomposition relations for several types of binary mixture systems shall be discussed in detail.

\section{Crystallization Temperature Diagram}

As seen from Figure 4, the crystallization temperature, read from the peak position in DSC diagram of Figure 3 , starts from nylon 66, decreases uniformly as the content of nylon 6 increases, and levels off after the content of nylon 6 exceeds $60 \%$. The crystallization becomes more protracted with dilution, ${ }^{15,16}$ so we can readily understand that crystallization temperature of nylon 66 in the mixtures decreases rapidly by adding the second component of nylon 6, since in this case nylon 6 plays the role of a diluent of amorphous polymer.

In Figure 6 , the heat of crystallization $\Delta H_{\mathrm{c}}$, obtained from the area under the crystallization profile in DSC diagram of Figure 3, is plotted against composition. In Figure 6, the heat of fusion $\Delta H_{\mathrm{m}}$, obtained from the area under the melting profile in DSC diagram of Figure 1, is plotted against composition. The composition dependence of $\Delta H_{\mathrm{c}}$ and $\Delta H_{\mathrm{m}}$ is very similar. The heat of melting is $45 \mathrm{cal} \mathrm{g}^{-113}$ for both crystals, and we can calculate the degree of crystallinity (see right vertical axis in Figure 6). For the mixtures with nylon 6 content of $0.2,0.3$, and 0.4 , we tried to separate $\Delta H_{\mathrm{m}}$ into contributions of two components based on the peak height. The results are shown in Figure 6. In the mixture in which nylon 6 content is less that $50 \%$, crystallization is governed by the formation of nylon 66 crystalline phase and the crystallization of nylon 6 is extremely restricted. Crystallization of nylon 66 is also inhibited somewhat by the presence of nylon 6 which acts as an amorphous polymer diluent, and total crystallinity is reduced. In fact, the degree of crystallinity of nylon 66 for the composition of nylon 6/nylon $66=$ $50 / 50$ is considerably reduced (about $30 \%$ less) in com-

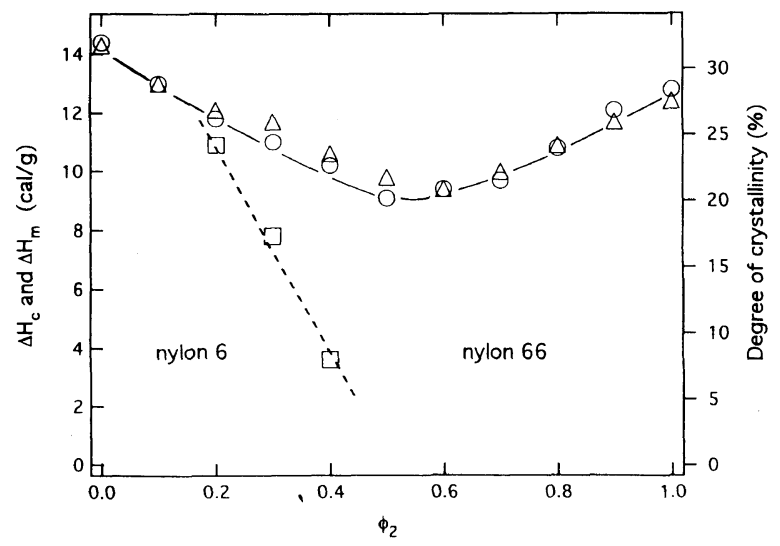

Figure 6. Composition dependence of the heat of melting, $\Delta H_{\mathrm{m}},(\triangle)$ and the heat of crystallization, $\Delta H_{c},(\bigcirc)$ obtained for the mixtures of nylon 6 and nylon 66. Right axis indicates the degree of crystallinity estimated using $45 \mathrm{cal} \mathrm{g}^{-1}$ for the heat of melting of the crystal of nylon 6 or nylon 66 . For mixtures with $\phi_{2}=0.2,0.3$, and 0.4 the heats of melting of nylon $6(\square)$ were estimated based on the peak height (see the text).

parison to pure nylon 66 . For the case in which nylon 6 is major, the crystallization of nylon 66 as a highermelting temperature component advantageously occurs. However, in this case, nylon 6 can crystallize in the space between nylon 66 crystal since the content of nylon 66 is not high. Thus, the crystallization of two components is inhibited by each other component. Consequently, the degree of crystallinity depends on the composition as shown in Figure 6. The crystallization mechanism in the mixture of two crystalline components will be discussed later.

For further investigation of the features of crystallization temperature diagram, we examined crystallization profile-composition relationships. The shape of each crystallization profile appeared at various crystallization temperatures on cooling in DSC diagrams for nylon $6 /$ nylon 66 mixtures is very similar, but the broadness of the profile changes depending on composition. The broadest profiles were observed in the intermediate region of the mixtures, i.e., $\phi_{2}=0.4-0.6$. We estimated the slope of increasing curve of the profile.

The time interval from the onset of crystallization to the point at which a maximum slope was observed was defined as a rate parameter implying the half-time of crystallization and designated as $t_{1 / 2}$. An estimation of $t_{1 / 2}$ can be made by differentiating the increasing curve and to read the maximum point of the differentiation curve, as shown in Figure 7 . The kinetic study by Magill ${ }^{17}$ used a simplified method and $1 / t_{1 / 2}$ instead of the rate constant $k$ in Avrami equation. In his paper, plots of $1 / t_{1 / 2} \quad v s$. crystallization temperature are presented. Similarly, in our case for nylon 6/nylon 66 mixtures, the plots of $t_{1 / 2} v s$. composition is shown in Figure 8. For the pure nylon 66 in our case, $1 / t_{1 / 2}=0.045 \mathrm{~s}^{-1}$, while in the literature of Magill, ${ }^{17}$ at the same crystallization temperature $230^{\circ} \mathrm{C}, 1 / t_{1 / 2}=0.073 \mathrm{~s}^{-1}$. Both values of $1 / t_{1 / 2}$ are of the same order, but quantitatively our value is smaller, about two thirds Magill's value. However, the comparative study on the quantitative bases is basically difficult, since we investigated the rate of crystal growth only as a preliminary study by using data obtained from the non-isothermal crystalliza- 


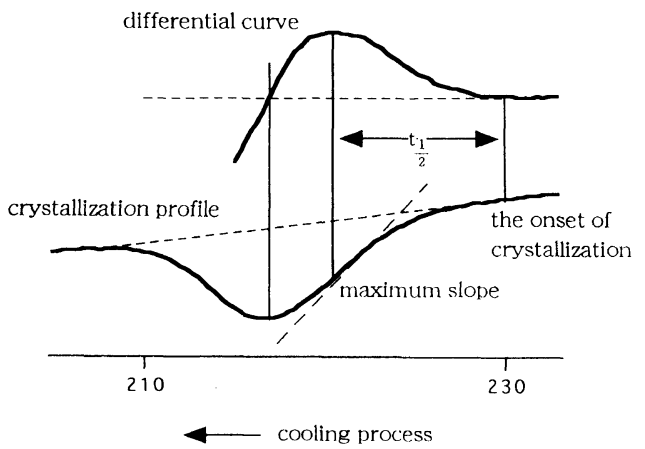

Figure 7. The estimation prodecure of $t_{1 / 2}$ is schematically illustrated. From the corresponding time interval $\delta T, t_{1 / 2}$ can be estimated by the relation $t_{1 / 2}(\mathrm{~s})=6 \cdot \delta T(\mathrm{deg})$, since the cooling rate is $10^{\circ} \mathrm{Cmin}^{-1}$.

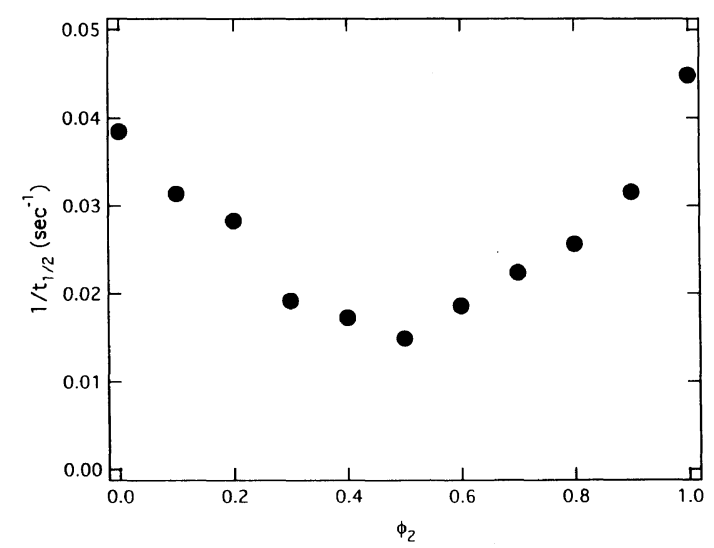

Figure 8. Relation between the parameter for the crystal growth rate introduced in this study and the composition of the mixture of nylon 6 and nylon 66.

tion during the course of cooling process in the measurement of DSC. Qualitative agreement of both values of $t_{1 / 2}$ mentioned above may be regarded satisfactory.

For case $\mathrm{A}$, the trend according to increase of nylon 6 content starting from pure nylon 66 , and $\mathrm{B}$, the trend according to increase of nylon 66 content starting from pure nylon 6 are discussed.

The temperature of the onset and peak position of crystallization profile shift downward according to nylon 6 content starting from nylon 66 . In terms of $1 / t_{1 / 2}$, the measure of crystal growth rate, it decreases from 0.045 $\mathrm{s}^{-1}$ of pure nylon 66 to $0.015 \mathrm{~s}^{-1}$ (almost one third) the mixture of nylon $6 /$ nylon $66=50 / 50$. Here, we must be cautious about the fact that the crystallization temperature dropped from $230^{\circ} \mathrm{C}$ of pure nylon 66 to $210^{\circ} \mathrm{C}$ of the mixture of nylon $6 /$ nylon $66=50 / 50$. This trend can be understood as the polymer-diluent effect of an amorphous nylon 6 in the binary mixtures of a crystalline-amorphous polymer. Although no quantitative study for the crystallization of the mixture of polyamides has been reported, in the kinetics study of PVDF/PMMA mixtures by Wang and $\mathrm{Nish}^{16}$ radial growth rate $G\left(\mu \mathrm{min}^{-1}\right)$ of PVDF spherulite decreased from $G=30$ of pure PVDF to $G=1.0$ (about $1 / 30$ ) in the mixture with $32.5 \%$ of PMMA at $140^{\circ} \mathrm{C}$. The crystal growth rate increases with quench depth $\Delta T$, the difference between the crystal melting temperature $T_{\mathrm{m}}$ and the crystallizing temperature $T_{\mathrm{c}}\left(\Delta T=T_{\mathrm{m}}-T_{\mathrm{c}}\right)$. In the paper of Magill, ${ }^{17} 1 / t_{1 / 2}$ of pure nylon 66 increased
Table II. Composition dependency of the onset and the peak position of the crystallization profile in the mixture of nylon 6 and nylon 66

\begin{tabular}{ccccc}
\hline $\begin{array}{c}\text { Composition } \\
{[\text { Component 2] }} \\
\phi_{2}\end{array}$ & $\begin{array}{c}\text { Temp at the onset } \\
\text { of crystallization }\end{array}$ & $\begin{array}{c}\text { Temp at the peak } \\
\text { position }\end{array}$ & $\begin{array}{c}\text { Rate parameter } \\
1 / t^{1 / 2}\end{array}$ \\
0 & ${ }^{\circ} \mathrm{C}$ & ${ }^{\circ} \mathrm{C}$ & $\mathrm{s}^{-1}$ \\
0.1 & 188 & 180 & 0.039 \\
0.2 & 187 & 178 & 0.032 \\
0.3 & 186 & 178 & 0.028 \\
0.4 & 190 & 177 & 0.019 \\
\hline
\end{tabular}

from $0.074 \mathrm{~s}^{-1}$ at $230^{\circ} \mathrm{C}$ to $0.22 \mathrm{~s}^{-1}$ at $210^{\circ} \mathrm{C}$. Thus, we may conclude crystal growth rate of nylon 66 in the mixture with nylon 6 to incur two effects: a dramatic decrease by the diluent effect of an amorphous nylon 6 and acceleration by the increase of $\Delta T$, and hence, we obtained the composition dependence of decreasing tendency of $1 / t_{1 / 2}$ for nylon 66 in the mixtures as the content of nylon 6 increases as shown in Figure 8.

The behavior of crystallization profile in the case B (see Table II) can be explained as follows. (i) The temperature of the onset of crystallization $\left(188^{\circ} \mathrm{C}\right.$ for pure nylon 6) remains almost the same in the mixture with small amount of nylon $66(10 \%, 20 \%$, and $30 \%)$ and shifts toward higher temperature when nylon 66 content becomes $40 \%$. (ii) The crystallization temperature (peak position) first decreases in the mixture with $10 \%, 20 \%$, and $30 \%$ of nylon 66 , then it remarkably shifts toward the higher temperature when the nylon 66 content proceed $40 \%$. (iii) The parameter for the crystal growth rate, $1 / t_{1 / 2}$, decreases from $0.039 \mathrm{~s}^{-1}$ of pure nylon 6 to $0.017 \mathrm{~s}^{-1}$ for the mixture with $40 \%$ nylon 66 (about $40 \%$ decrease). This tendency of $1 / t_{1 / 2}$ decreasing with the mixing amount (item (iii)) is the same as in case A. However, the tendencies of the temperature of the onset and the peak position of crystallization ((i) and (ii)) are completely different. We explain this behavior based on the following model. It can be thought that nylon 66, the higher-melting temperature component, forms a primary nucleus in cooling from the molten state even if nylon 66 content is small. In the mixture where nylon 66 content is small, nylon 6 molecules can start easily to crystallize on the surface of the primary nucleus of nylon 66. However, since the crystal growth rate of the proceeding process should be based on the secondary nucleation mechanism, it decreases compared to pure nylon 6 . When increase of nylon 66 content, $1 / t_{1 / 2}$ decreases, while the broadness of the crystallization profile increases. As a result of the broadening of the profile while the onset temperature remains same, the peak position of the profile shifts downward. In the region of small content of nylon 66 , the dominant component of the crystallization is nylon 6 . However, for the case of more than $40 \%$ nylon 66 content, the dominant component moves to nylon 66, and both the onset and the peak position of the crystallization profile shift toward the higher temperature significantly. 


\section{CONCLUSIONS}

Measurements of DSC for binary mixtures of nylon 6 and nylon 66 (prepared from well-mixed solution) were performed, and the following conclusions were obtained.

(1) During solidification of the mixture samples on cooling from the melt, nylon 66 , the higher melting temperature component, crystallizes preferably, while the crystallization of nylon 6 is remarkably restricted, especially when nylon 6 is less than $50 \%$. Two melting peaks of nylon 6 and nylon 66 are observed, in the limited range; $\phi_{2}=0.1-0.4$, where $\phi_{2}$ is the fraction of nylon 66 .

(2) A considerable reduction of melting temperature $T_{\mathrm{m} 2}$ of nylon 66 was recognized over the whole range of composition, probably due to the diluent effect caused by nylon 6 . Analysis of data in terms of Nishi-Wang equation indicated the polymer-polymer interaction parameter to be $\chi=-0.56$ and $B=-5.1 \mathrm{cal} \mathrm{cm}^{-3}$ at $250^{\circ} \mathrm{C}$. The contribution from the entropy term to the parameter $\chi$ is significant.

(3) In DSC diagram on cooling, the crystallization temperature $T_{\mathrm{c}}$ read at the peak position, starts from nylon 66, decreases in the mixtures as the content of nylon 6 increases and levels off at $\phi_{2}=0.3-0.1$. This level-off behavior can be attributed to the fact that nylon 6 molecules easily start to crystallize on the surface of primary nucleus of nylon 66 which has formed preferably.

(4) The total degree of crystallinity including both nylon components estimated from the area under the crystallization profile of DSC diagram shows a remarkable decrease, by $25-30 \%$, in the intermediate range of composition, i.e., $0.4<\phi_{2}<0.6$.

(5) The slope of increasing curve of each profile on cooling process, as a measure of the rate of crystal growth, decreases remarkably in the mixtures, especially in the intermediate region of composition, and becomes to be $1 / 2-1 / 3$ compared to pure nylon 6 or nylon 66 .

Acknowledgment. This work was supported by the international joint research grant of NEDO (New Energy and Industrial Technology Development Organization). The authors are indebted to Ms. Yasuko Suzuki (Unitika Research Laboratories Inc.) for her assistance with the experimental work.

\section{REFERENCES}

1. K. Matsumura, T. Shimomura, T. Matsuda, and M. Hirami, to be submitted to Polym. J.

2. T. Shimomura, T. Matsuda, and M. Hirami, to be submitted to Polym. J.

3. P. J. Flory, "Principles of Polymer Chemistry," Cornell University, Ithaca, N.Y., 1953, Chapter XIII-2.

4. L. Mandelkern, "Crystallization of Polymers," McGraw-Hill, New York, N.Y., 1963, Chapter 3.

5. T. Nishi and T. T. Wang, Macromolecules, 8, 909 (1975).

6. L. Mandelkern, in "Comprehensive Polymer Science, Vol. 2, Polymer Properties," C. Booth and C. Price, Ed., Pergamon Press, New York, N.Y., 1989, Chapter 11.

7. R. S. Stein, in "Crystallization of Polymers," M. Dosiere, Ed., Kluwer Academic Publishers, Dordrecht, The Netherlands, 1993, pp $421-435$.

8. L. Mandelkern, R. G. Alamo, G. D. Wignall, and F. C. Stehling, TRIP, 4, 377 (1996).

9. H. Mitomo and H. Tonami, Chem. High Polym., 27, 134 (1970).

10. E. S. Ong, Y. Kim, and H. L. Williamson, J. Appl. Polym. Sci., 31, 376 (1986).

11. F. Rybnikar and P. H. Geil, J. Appl. Polym. Sci., 46, 797 (1992); ibid., 49, 1175 (1993).

12. P. J. Flory, Disc. Faraday Soc., 49, 7 (1970).

13. Reference 4, Chapter 5-2, p 120.

14. M. Hirami and T. Matsuda, Polym. J., 31, 801 (1999).

15. Reference 4, Chapter 8-7, p 273.

16. T. T. Wang and T. Nishi, Macromolecules, 10, 421 (1977).

17. J. H. Magill, Polymer, 2, 221 (1961). 